\title{
Implementación de los sistemas de planificación de recursos empresariales (ERP) en las organizaciones desde la coevolución
}

\author{
Implementation of Enterprise Resource Planning (ERP) \\ Systems in Organizations Since Coevolution
}

\author{
Andrés Camilo Rivera-Silva $₫$, Rafael Esteban Vargas-Reyes ${ }^{2}$, \\ Luz Esperanza Bohórquez-Arévalo ${ }^{3}$
}

1 Universidad Distrital Francisco José de Caldas, Bogotá, Colombia
${ }^{2}$ Universidad Distrital Francisco José de Caldas, Bogotá, Colombia
${ }^{3}$ Universidad Distrital Francisco José de Caldas, Bogotá, Colombia

Carrera 7 N. ${ }^{\circ}$ 40-53, Universidad Distrital Francisco José de Caldas, Facultad de Ingeniería. Correo electrónico: acriveras@correo.udistrital.edu.co.

Recibido: 5 de agosto del $2017 \quad$ Aprobado: 19 de noviembre del 2017 Disponible en línea: 1 de enero del 2018

How to cite this article: A. C. Rivera-Silva, R. E. Vargas-Reyes y L. E. Bohórquez-Arévalo, "Implementación de recursos empresariales (ERP) en las organizaciones desde la coevolución”, Revista Ingeniería Solidaria, vol. 14, no. 24, pp. 15, enero 2018. doi: https://doi.org/10.16925/in.v14i24.2161

\section{Resumen}

Introducción: el artículo de revisión bibliográfica es producto de la investigación "Implementación de los sistemas de planificación de recursos empresariales (ERP) en las organizaciones desde la coevolución", realizada durante el 2016 y el 2017 en la Facultad de Ingeniería Industrial de la Universidad Distrital Francisco José de Caldas.

Problema: según diversas investigaciones, las implementaciones de ERP han resultado fallidas y generan pérdidas empresariales.

Objetivo: diseñar una propuesta de estudio para que la implementación de ERP resulte exitosa en las organizaciones.

Metodología: se inicia por revisar la literatura relacionada con implementación de ERP en las organizaciones e identificar que el 70 \% de las empresas no obtuvieron los resultados esperados. Se plantea que la adopción de ERP se puede comprender como un proceso coevolutivo basado en la interacción entre los empleados a través de redes de afiliación formales e informales.

Resultados: se propone iniciar por la identificación de las personas que tienen mayor potencial de influir sobre las demás, de manera que se inicie un proceso de propalación de información que circula desde los nodos más influyentes hacia aquellos con los que interactúan.

Conclusión: la comprensión del cambio como proceso de coevolución en redes formales e informales da pie a la opción de discutir y repensar la forma en que están organizadas las empresas.

Originalidad: hasta el momento no se ha abordado la implementación de este tipo de plataformas desde este punto de vista.

Limitaciones: la propuesta se encuentra en fase conceptual, sin embargo, aún no se cuenta con investigación empírica que permita su validación.

Palabras clave: coevolución, gestión del cambio, implementación, redes formales e informales, sistema de planificación de recursos empresariales (ERP). 


\title{
Implementation of Enterprise Resource Planning (ERP) Systems in Organizations Since Coevolution
}

\author{
Abstract \\ Introduction: The literature review article derives from the research "Implementation of enterprise resource planning \\ (ERP) systems in organizations since coevolution" conducted in 2016 and 2017 at the School of Industrial Engineering, \\ Universidad Distrital Francisco José de Caldas.
}

Problem: According to various research papers, ERP implementations have failed and generate business losses.

Aim: To design a study proposal for the successful implementation of ERP in organizations.

Methods: We started from a review of the literature related to ERP implementation of in organizations and identified that $70 \%$ of companies did not obtain the expected results. It is argued that ERP adoption can be understood as a coevolutionary process based on the interaction among employees through formal and informal affiliation networks.

Results: It is proposed that individuals who have the greatest potential to influence others are identified in order to initiate an information dissemination process that flows from the most influential nodes to those with which they interact.

Conclusion: The understanding of change as a coevolution process in formal and informal networks provides an opportunity to discuss and rethink the way in which companies are organized.

Originality: To date, the implementation of this type of platform has not been addressed from this point of view.

Limitations: The proposal is in the conceptual phase, but there is still no empirical research that allows its validation.

Keywords: coevolution, change management, implementation, formal and informal networks, enterprise resource planning (ERP) system.

\section{Implementação dos sistemas de planejamento de recursos empresariais nas organizações (ERP) a partir da coevolução}

\section{Resumo}

Introdução: este artigo de revisão bibliográfica é produto da pesquisa "Implementação dos sistemas de planejamento de recursos empresariais (ERP) nas organizações a partir da coevolução", realizada durante 2016 e 2017, na Faculdade de Engenharia Industrial da Universidad Distrital Francisco José de Caldas.

Problema: segundo diversas pesquisas, as implementações de erp fracassam e geram perdas empresariais.

Objetivo: desenhar uma proposta de estudo para que a implementação de ERP seja bem-sucedida nas organizações.

Metodologia: inicia-se com a revisão da literatura relacionada com implementação de ERP nas organizações e identificação de que 70 \% das empresas não atingiram os resultados esperados. Propõe-se que a adoção de ERP pode ser compreendida como um processo coevolutivo baseado na interação entre os empregados por meio de redes de afiliação formais e informais.

Resultados: propõe-se iniciar pela identificação das pessoas que têm maior potencial de influenciar sobre as demais, de maneira que se inicie um processo de propagação de informação que circula desde os nodos mais influentes até aqueles com os quais interagem.

Conclusão: a compreensão da mudança como processo de coevolução em redes formais e informais propicia discutir e repensar a forma em que as empresas estão organizadas.

Originalidade: até o momento, não tem sido abordada a implementação desse tipo de plataformas sob esse ponto de vista.

Limitações: a proposta encontra-se em fase conceitual, mas ainda não se conta com pesquisa empírica que permita sua validação.

Palavras-chaves: coevolução, gestão da mudança, implementação, redes formais e informais, sistema de planejamento de recursos empresariais (ERP). 


\section{Introducción}

El artículo "La implementación de ERP en las organizaciones empresariales como proceso de cambio desde la coevolución: una propuesta de estudio" es producto de la investigación realizada durante el 2016 y el 2017 a cargo de los estudiantes Andrés Camilo Rivera y Rafael Esteban Vargas, con la alineación de la docente Ph.D. Luz Esperanza Bohórquez, como proyecto de grado de la Facultad de Ingeniería Industrial de la Universidad Distrital Francisco José de Caldas. Se conoce como enterprise resource planning (ERP) al conjunto de módulos de software integrados y una base de datos central que permite gestionar el uso eficiente y eficaz de los recursos (materiales, recursos humanos, finanzas, etc.) mediante la automatización y la incorporación de los procesos de negocio, intercambio de datos en toda la empresa y el acceso a la información en el entorno en tiempo real [4], [5]. Estas plataformas brindan soporte a los diferentes procesos empresariales y generan ventaja competitiva gracias a los flujos de información que permiten.

Desde su aparición en la década de los noventa las ERP han demostrado diversas ventajas que pueden comprenderse en diferentes categorías: en el área operativa trae beneficios en términos de eficiencia de los procesos empresariales y las transacciones en la empresa; en el área gerencial los beneficios se manifiestan en los flujos de información disponibles para la gestión, la toma de decisiones y el control del rendimiento de la organización. En cuanto a la estrategia, el uso de ERP es determinante en términos de diferenciación, costo, innovación, crecimiento y alianzas con los grupos de interés. La adecuada adopción y uso de ERP logra consolidar efectivamente la infraestructura de tecnologías informáticas y facilita los flujos de información. En cuanto a la condición organizativa, los beneficios se manifiestan en mejor desempeño del recurso humano, así como en la cultura de trabajo dentro de la empresa [6].

\subsection{Antecedentes de investigación en implementación de ERP}

Las notorias ventajas que pueden generar ERP han llevado a que un amplio número de empresas en el mundo decidan adoptar este tipo de tecnología. Sin embargo, en muchos casos no se cumplen los objetivos de la implementación. Según la investigación de Chou y Chang, en el 2008 un tercio de los proyectos de implementación de dichas plataformas no tienen éxito, y entre el 60\% y el 70\% de los casos de aplicación fracasan en la obtención de los beneficios esperados [7], lo cual acarrea pérdidas económicas significativas. En la tabla 1 se presenta un recuento de diferentes casos fallidos de implementación de ERP [8]-[11], al que se suman los resultados de la investigación desarrollada por Kimberling [1]. En esta recopilación se resaltan las consecuencias y los efectos negativos que se obtienen debido a una implementación fallida de ERP.

Tabla 1. Casos fallidos de implementación de ERP y consecuencias en el desempeño empresarial

\begin{tabular}{lcl}
\hline Nombre de la organización & Año & \multicolumn{1}{c}{ Fallas y problemas en los proyectos ERP } \\
\hline $\begin{array}{l}\text { National Health Service } \\
\text { (NHS), Reino Unido }\end{array}$ & $\mathbf{2 0 1 1}$ & $\begin{array}{l}\text { Después de gastar unos } 12 \text { 000 millones de libras esterlinas (USD 18 700 } \\
\text { millones), NHS abandonó el proyecto que tenía como objetivo centralizar los } \\
\text { registros de salud electrónicos de sus ciudadanos. }\end{array}$ \\
\hline $\begin{array}{l}\text { CityTime Payroll System } \\
\text { project, Nueva York, EE. uU. }\end{array}$ & $\mathbf{2 0 1 1}$ & $\begin{array}{l}\text { El proyecto fracasó debido a los excesos de costos y de presupuestos que } \\
\text { pasaron de usD 63 millones a una cantidad estimada de usD 760 millones, y } \\
\text { una investigación criminal. }\end{array}$ \\
\hline Ingram Micro Australia & $\mathbf{2 0 1 1}$ & $\begin{array}{l}\text { El problema con la implementación de sAP en Ingram Micro llevó a una } \\
\text { caída significativa en su ingreso neto dos veces en el 2011. }\end{array}$ \\
\hline $\begin{array}{l}\text { Montclair State University, } \\
\text { Nueva Jersey, EE. UU. }\end{array}$ & $\mathbf{2 0 1 1}$ & $\begin{array}{l}\text { La implementación de PeopleSoft en la Universidad Estatal de Montclair } \\
\text { enfrentó problemas que llevaron a la Universidad a presentar una demanda } \\
\text { contra Oracle por la implementación fallida. }\end{array}$ \\
\hline ParknPool, EE. UU. & $\mathbf{2 0 1 1}$ & $\begin{array}{l}\text { La empresa de vendedores de muebles demandó a Epicor por el fallido } \\
\text { proyecto ERP. }\end{array}$ \\
\hline
\end{tabular}


(viene)

\begin{tabular}{|c|c|c|}
\hline Nombre de la organización & Año & Fallas y problemas en los proyectos ERP \\
\hline $\begin{array}{l}\text { Marin County, California, } \\
\text { EE. UU. }\end{array}$ & 2011 & $\begin{array}{l}\text { Marin County presentó una demanda contra Deloitte Consulting y SAP sobre } \\
\text { un proyecto ERP fallido. }\end{array}$ \\
\hline $\begin{array}{l}\text { Whaley Foodservice } \\
\text { Repairs, Carolina del Sur, } \\
\text { EE. UU. }\end{array}$ & 2011 & $\begin{array}{l}\text { Epicor fue demandado por la compañía de equipos de cocinas comerciales } \\
\text { por un proyecto que costó a la compañía más de cinco veces la cantidad } \\
\text { original estimada de USD } 190000 .\end{array}$ \\
\hline State of Idaho, EE. UU. & 2011 & $\begin{array}{l}\text { Idaho enfrentó problemas debido a defectos de diseño y otros problemas que } \\
\text { provocaron varios retrasos en los pagos y los procesamientos de reclamos } \\
\text { defectuosos después de instalar un nuevo sistema proporcionado por Unisys. } \\
\text { El estado podría sufrir pérdidas de millones de dólares debido a las reclama- } \\
\text { ciones defectuosas de Medicaid. }\end{array}$ \\
\hline $\begin{array}{l}\text { The Victorian Order of } \\
\text { Nurses, Nueva Escocia, } \\
\text { Canadá }\end{array}$ & 2011 & $\begin{array}{l}\text { La implementación del sistema de nómina de pago de sAP resultó en la emi- } \\
\text { sión de salarios defectuosos a las enfermeras durante al menos seis meses. }\end{array}$ \\
\hline
\end{tabular}

\begin{tabular}{|c|c|c|}
\hline Lumber Liquidators & 2010 & Se encontraron problemas con el sistema SAP. \\
\hline Dillard's, Inc. & 2010 & La implementación no logró satisfacer las expectativas de los clientes. \\
\hline $\begin{array}{l}\text { Ferazzoli Imports of New } \\
\text { England }\end{array}$ & 2009 & $\begin{array}{l}\text { El sistema de Epicor no cumplió con las expectativas del cliente como se } \\
\text { había prometido. }\end{array}$ \\
\hline MillerCoors & 2016 & $\begin{array}{l}\text { La cervecera MillerCoors, con sede en Chicago, demandó a la cuarta mayor } \\
\text { firma de servicios de tecnología de la India HCL Technologies y su división } \\
\text { estadounidense por USD } 100 \text { millones de dólares, por incumplimiento de } \\
\text { contrato en un proyecto para implementar el software comercial sAP. }\end{array}$ \\
\hline Target Canada & 2013 & $\begin{array}{l}\text { La cadena de suministro de la compañía se colapsó y los investigadores } \\
\text { rastrearon rápidamente la falla hasta esta información supuestamente nueva, } \\
\text { la cual estaba plagada de errores. }\end{array}$ \\
\hline PG\&E & 2016 & $\begin{array}{l}\text { La base de datos de Pacific Gas and Electric, con detalles de más de } 47000 \\
\text { computadoras, máquinas virtuales, servidores y otros dispositivos quedó } \\
\text { expuesta y completamente abierta a visualización, sin nombre de usuario o } \\
\text { contraseña requerida; esta información quedó expuesta como resultado de } \\
\text { un despliegue de ERP. }\end{array}$ \\
\hline Nike & 2001 & $\begin{array}{l}\text { En el } 2000 \text { y el 2001, Nike gastó USD } 400 \text { millones de dólares actualizando su } \\
\text { sistema de cadena de suministro y la implementación de ERP. Se obtuvo un } \\
\text { descenso del } 20 \% \text { en sus acciones, UsD } 100 \text { millones de dólares en ingresos } \\
\text { perdidos y una miríada de demandas colectivas. }\end{array}$ \\
\hline Cubic Corporation & 2017 & $\begin{array}{l}\text { Cubic Corporation invirtió en un sistema ERP con el fin de optimizar las } \\
\text { operaciones y mejorar la rentabilidad. Para el } 2017 \text { los costos de software e } \\
\text { implementación superan los USD } 61 \text { millones y ascienden. }\end{array}$ \\
\hline
\end{tabular}

Fuente: adaptado de [1], [8]-[11]

Las fallas en los procesos de implementación de ERP ha llevado a que diversos investigadores enfoquen su atención en la búsqueda de alternativas que permitan disminuir el porcentaje de fracaso en la adopción de este tipo de plataformas. Las investigaciones se han orientado, de una parte, al desarrollo de metodologías y estrategias para la implementación de ERP, y de otra al estudio de las causas de éxito y fracaso en los procesos de implementación. Sin embargo, en los dos casos el foco central de atención ha sido cómo garantizar la adopción de la nueva plataforma tecnológica.

De manera general, las metodologías de implementación se han orientado al diseño de rutas óptimas que faciliten la adopción de la plataforma tecnológica. Dentro de las principales actividades que se han considerado en dichas metodologías se encuentran: la planificación del proyecto (calendario, recursos, etc.), el estudio y la comprensión de los procesos empresariales actuales y los flujos 
de información y reingeniería de procesos que permitan reducir actividades redundantes, identificación de necesidades de personalización de sistema, configuración, pruebas piloto, formación del usuario, instalación y puesta en marcha, migración de datos, ajustes, corrección de errores y actualización.

Dentro de las investigaciones orientadas a la identificación de las causas de éxito y fracaso se encuentra la desarrollada por Leyh en el 2014 [2]. En esta se realiza un recuento con 320 fuentes diferentes en la que se busca resumir cuáles son los factores críticos de éxito que más se repiten en la implementación de ERP según diferentes autores. Los resultados indicaron que aspectos tales como el soporte de la alta dirección, la gestión de proyectos, el entrenamiento de usuario, la gestión del cambio, el equipo del proyecto, la comunicación, la participación del usuario final y de los stakeholders, así como la aceptación o resistencia del sistema ERP se encuentran dentro de los factores decisivos que dificultan la apropiación de la plataforma tecnológica en las empresas.

A partir de lo anterior se puede afirmar que, tal como lo plantean K. Zhu, K. Kraemer y S. Xu, el principal desafío de la implementación de ERP no se trata de cómo instalar ERP, sino de cómo hacer que el sistema se utilice en la actividad cotidiana de la empresa [12]. Es en este punto que la presente investigación plantea que la implementación de ERP en las organizaciones puede considerarse como un proceso de cambio comprendido desde la biología, y puntualmente desde la coevolución.

La coevolución, desde la biología, reconoce que el comportamiento del colectivo y, de manera amplia, el cambio que emerge en el sistema surge de la interacción no proporcional entre las partes que lo conforman. Esta base evolutiva cuyos planteamientos se alejan del reduccionismo y, en general, del mecanicismo, se encuentra en consonancia con una conceptualización más amplia y reciente de la tecnología [13].

El presente artículo presenta los aspectos conceptuales que soportan el diseño de un modelo para la implementación de ERP que promuevan su uso generalizado en las actividades cotidianas de las empresas, es decir, la adopción de ERP se comprende como un proceso evolutivo que debe redundar en un comportamiento colectivo que de manera permanente se transforma. La propuesta exalta la importancia de la interacción entre las personas y la ausencia de factores que determine el comportamiento del sistema como fuentes centrales que explican el comportamiento colectivo en el uso de ERP.

Es importante señalar que la biología, en la ciencia moderna, se ha constituido en la principal fuente de teorías, modelos y, en general, de conocimiento para la comprensión de fenómenos y sistemas caracterizados por la emergencia de comportamientos colectivos a partir de la interacción entre agentes. De esta manera, tal como lo planteó Andrade en 1996, el desarrollo científico tanto en el contexto natural como social ha sido el resultado de múltiples interacciones, en las que los modelos o modos propuestos por una disciplina influyen los modelos o modos de ver las otras [14].

\subsection{La coevolución y la pertinencia de su aplicación en contextos diferentes a la biología}

El concepto de coevolución según Thompson tiene su origen en la biología y se refiere a los cambios recíprocos en especies interactuantes [15]. Desde esta perspectiva, dos o más especies que se relacionan entre sí cambian en respuesta a cómo cambia la otra; es decir, cada cual está pendiente de los agentes que se encuentran a su alrededor, tales como: depredador-presa y parásito-hospedador, especies competidoras o especies mutualistas. La reciprocidad es la característica definitoria de las interacciones coevolutivas en general. No solo la adaptación por un jugador debe favorecer el cambio en su oponente, sino que la adaptación del oponente también debe generar la selección y la respuesta evolutiva en el primer jugador [16]. Un ejemplo se encuentra en la relación entre un parásito y su anfitrión; tanto el parásito como el huésped están involucrados en una carrera de armamento entre sí. Existe una presión sobre el huésped a evolucionar para volverse resistente al parásito, así como una presión sobre el parásito para desarrollar maneras de hacer frente a la resistencia del huésped. Ambas especies necesitan cambiar genéticamente para mantenerse al día con los cambios en las otras especies [17].

Esta interacción que se da entre las especies es la misma que se da entre las empresas: entre empresas competidoras, con sus clientes, con sus proveedores y entre las personas al interior de la organización. 


\subsection{Coevolución en el contexto empresarial}

Desde la década de los setenta del siglo xx diversos teóricos introdujeron la perspectiva evolutiva con el propósito de ofrecer una alternativa a la explicación de cómo las organizaciones se adaptan bien a los cambios en su entorno. De acuerdo con Dimmick [18], los estudiosos de la organización Hannan y Freeman en 1977 [19], Aldrich en 1979 [20], Weick en 1979 [21], y McKelvey en 1982 [22], se dieron cuenta de cómo la acción intencional anticipada desarrollada por Andrews en 1971 [23] y Child en 1972 [24] no es la única manera de explicar los cambios en las organizaciones. De hecho, según Murmann existen otras vías para explicar el cambio, las cuales se encuentran en la interacción entre entidades distintas (variación), en la presión del entorno por eliminar la entidad mal adaptada (selección), así como en la supervivencia a través del tiempo de las entidades adaptadas (retención) [25].

La idea de la coevolución cobra importancia debido a la percepción generalizada según la cual los entornos son cada vez más rápidos [26], más competitivos [27] y más turbulentos [28], de modo que crean una mayor oportunidad para influir en las características del entorno macro [29] y permiten así a la organización adaptarse y sobrevivir.

Sin embargo, aunque mucho trabajo ha señalado la coevolución en el contexto empresarial y se ha afirmado su importancia, es escaso el que precisa cómo las organizaciones coevolucionan de acuerdo con el impacto de los principios de variación, selección y retención característicos del cambio evolutivo [25].

Lo que aparta a la teoría coevolutiva de la explicación evolutiva estándar es que la posibilidad de influir no solo corre del ambiente hacia la organización, sino también de la organización al medio ambiente y de manera interna [29]. Es decir, la coevolución no es exclusiva de la interacción con el entorno externo, sino que también surge de la interacción entre las partes que conforman el sistema. De manera amplia una organización coevolutiva es aquella que se modifica a sí misma, modifica el entorno con el que interactúa y se adapta a las condiciones cambiantes del medio en el que se encuentra.

En esta línea, investigaciones hechas por Yates en 1993 [30], Nelson en 1994 [31], Rosenkopf y
Tushman en 1994 [32], Murmann en el 2003 [29], Henderson y Stern en el 2004 [33] y Funk en el 2009 [34] plantean que la tecnología cambia mientras la industria que la usa o produce sufre también algún cambio. Un ejemplo concreto es el Internet: las empresas no solo se ven afectadas por las tecnologías de Internet, sino que casos como Microsoft, Apple, Google y similares también tienen un impacto dramático sobre cómo se desarrollan las tecnologías de Internet.

Lo anterior ha llevado a afirmar que los sistemas caracterizados por altos niveles de interacción internos y con el entorno - como es claro que lo son las organizaciones empresariales- son sistemas coevolutivos alejados del equilibrio. El comportamiento en estos sistemas no puede explicarse a partir del comportamiento de las partes. La interdependencia entre los diferentes agentes que los conforman, así como la interacción de estos con el entorno permite que el sistema se organice como un todo que cambia de manera permanente. Los sistemas alejados del equilibrio constituyen la regla y no la excepción de un conjunto suficientemente amplio de sistemas sociales [35]-[37].

\subsection{Antecedentes de investigación de uso de conceptos biológicos en campos empresariales}

Existen amplios antecedentes del impacto del enfoque biológico en la ingeniería y la gestión tecnológica, principalmente en la imitación del comportamiento de lo vivo (adaptabilidad, evolución, cambio, autoorganización, etc.), y otros elementos biológicos que han servido de base para el planteamiento de metáforas y analogías en el desarrollo de conceptos, herramientas y técnicas para una mejor comprensión de la realidad [13], [38]. Brezocnik y Balic en el 2001, a partir de su investigación, aportan un desarrollo de sistemas de manufactura flexible y sistemas inteligentes con características tales como la autoorganización, la adaptación eficiente y la evolución [39]. Ueda, Vaario y Ohkura, en 1997, a su vez plantean la conceptualización e implementación de sistemas biológicos de manufactura cuyo modelamiento se inspira en el comportamiento, los atributos y las estructuras de los organismos vivos [40]. Asimismo, la gestión de sistemas de integración empresarial desde una perspectiva biológica en Montoya [41], el impacto de los conceptos biológicos y la imitación del comportamiento de 
organismos vivos en el incremento de la productividad de los sistemas de manufactura en Otálora [42], y la valoración tecnológica en las organizaciones en Jiménez [13], entre otros.

La coevolución se encuentra en clara oposición con el enfoque reduccionista y determinista que caracteriza el paradigma tradicional para la comprensión de las organizaciones empresariales [43], [44]. Desde la perspectiva coevolutiva se reconoce la emergencia de comportamientos colectivos a partir de la interacción adaptativa entre las partes, es decir, pierde validez el enfoque reduccionista pues no es posible deducir las propiedades de todo a partir de las propiedades de los componentes.

La coevolución proporciona un marco de estudio orientado a promover el uso de ERP en las actividades cotidianas de la empresa que busquen su implementación, ya que reconoce que la adopción (internalización de la plataforma) por parte de todos los miembros de la empresa es un proceso de cambio continuo, el cual no puede abordarse desde el determinismo prevaleciente en la visión tradicional de la organización como sistema estático e invariante. Desde la perspectiva coevolutiva no es posible asumir que el éxito en la implementación de ERP está determinado por una o algunas variables claves. Por el contrario, reconoce que la adopción de ERP se convierte en un comportamiento colectivo en razón a la interacción adaptativa entre las diferentes personas que integran el sistema.

\section{Metodología}

Para el desarrollo del presente artículo se parte de identificar como problema el alto porcentaje de fracaso en la implementación de ERP en las organizaciones. Esto gracias al estudio de la investigación realizada por Chou y Chang, que evidencia cómo un tercio de los proyectos de implementación de dichas plataformas no tiene éxito, y entre el 60\% y el $70 \%$ de los casos de aplicación fracasan en la obtención de los beneficios esperados [7]. Además, se tiene en cuenta la recopilación realizada por Leyh en el 2014 de 320 fuentes diferentes de investigación, en la que busca resumir cuáles son los factores críticos de éxito que más se repiten en la implementación de ERP según diferentes autores [2]. También se parte de la investigación de Kimberling, en la cual se detallan diferentes casos reales de implementación de plataformas ERP fallidas que generaron importantes pérdidas económicas y empresariales [1].

Luego, al tener en cuenta que la expresión cambio tiene estrecha relación con el termino evolución, se lleva a cabo una revisión bibliográfica con el propósito de encontrar en la naturaleza cuál es el factor fundamental en el éxito de la evolución de las especies. De esta manera, se identifica la coevolución como característica propiciadora de cambio a partir de la relación que existe entre las especies y de acuerdo con sus comportamientos colectivos.

Lo anterior da lugar a entender la coevolución como alternativa para la comprensión de la emergencia de comportamientos colectivos, así como a la pertinencia de su aplicación en contextos diferentes a la biología.

Con base en los altos niveles de interacción interna y externa que se tiene en las organizaciones - similar al comportamiento en la vida silvestre-, se investigan las cualidades de las redes formales e informales y se detecta cómo a partir de allí es posible enfrentar el problema de internalización de ERP para cada uno de los colaboradores de la organización.

La propuesta se desarrolla a partir de la premisa según la cual el uso generalizado de ERP en las actividades cotidianas de la empresa puede comprenderse como la emergencia de un comportamiento colectivo en un sistema caracterizado por altos niveles de interacción interna y externa. Existen lazos sociales que surgen en los empleados y denotan relaciones de amistad, compañerismo, etc. Las redes informales tienen la ventaja de fomentar la existencia de vínculos fuertes entre los empleados, los cuales refuerzan la comunicación, la confianza, la generación de ideas creativas, promueven la innovación y permiten a las organizaciones que operan en entornos altamente cambiantes responder de manera flexible y dinámica [45].

Se propone que la internalización del uso de ERP en las actividades cotidianas de la empresa debe partir, en primera instancia, de la identificación de las personas que tienen el mayor potencial de influir sobre las demás, siempre y cuando se consideren criterios de grado, intermediación y cercanía.

De esta manera, la segunda parte de la propuesta se basa en que la internalización de ERP debe iniciarse como un proceso de propalación de información acerca de las características, las ventajas y las limitaciones, entre otros aspectos de ERP, que circulan desde los nodos más influyentes hacia 
aquellos con lo que interactúan. A medida que la información sobre el buen uso y las cualidades de ERP se internaliza entre los protagonistas de la operación, la resistencia al cambio disminuye y los resultados del proceso deben ser cada vez mejores.

Con ayuda del programa Net Logo, desarrollado por U. Wilensky en 1999, se realizan simulaciones que evidencian la efectividad del flujo de información a partir de nodos (personas) que, por diferentes razones, pueden ejercer altos niveles de influencia sobre aquellos con los que interactúan.

Los resultados demuestran cómo a medida que pasa el tiempo la información se propaga entre todas las personas al interior de la organización, de modo similar a lo que ocurre con un virus o un rumor. El éxito está en garantizar este flujo de información en todo momento y en que las personas escogidas como fuentes estén alineadas con las estrategias de la dirección y vayan en pro de lo que se quiere lograr.

La investigación al respecto en organizaciones empresariales se encuentra en etapas tempranas, $y$ a la fecha no existe un modelo o una propuesta que facilite aprovechar la coevolución de las redes inter e intraorganizacionales. La presente investigación se encuentra en fase conceptual y presenta limitaciones relacionadas con la carencia de investigación empírica que permita su validación con información del mundo real.

En la parte final del artículo se despliegan las conclusiones y se plantea la discusión que da pie a un avance de la propuesta y a futuras líneas de investigación.

\section{Resultados}

La investigación demuestra que, si bien a la fecha existen metodologías para la adopción de ERP, no se ha definido de manera concreta cómo gestionar correctamente su implementación. Tampoco se ha relacionado este éxito con el que se da en la coevolución entre agentes que participan de forma simultánea para lograr un cambio exitoso en la naturaleza. Dado que no se ha profundizado el éxito de la gestión del cambio organizacional desde el campo biológico, este artículo ofrece una propuesta en la que se enlazan estos dos contextos, y demuestra a su vez la importancia de las relaciones formales e informales que se dan entre los agentes de una organización.

\subsection{Propuesta de estudio para la adopción de ERP en la organización desde la perspectiva coevolutiva}

La propuesta parte de la premisa de que el uso generalizado de ERP en las actividades cotidianas de aquellas empresas que decidan implementarlo se puede comprender como la emergencia de un comportamiento colectivo en un sistema caracterizado por altos niveles de interacción interna y externa [46]. La interacción interna hace referencia a los vínculos entre los empleados que posibilitan la capacidad de influir y ser influido por otros con los que se interactúe. Por su parte, la interacción externa enfoca los vínculos de los empleados y los miembros de la organización con otros que no hacen parte de la empresa, los cuales incluyen clientes, proveedores, competidores, amigos y familiares, entre otros individuos que pueden influir y ser influidos.

Las redes externas facilitan el acceso a mayores fuentes de recursos (información, conocimiento, experiencia, capital, etc.). Las redes internas pueden generar intercambios positivos y beneficiosos de los recursos interpersonales como, por ejemplo, la satisfacción laboral (el intercambio de los recursos aumenta la percepción de apoyo del compañero de trabajo), y el arraigo en el empleo (relacionado con el sentimiento de vinculación y gusto por el trabajo) [47].

En las organizaciones empresariales, y de manera amplia en los sistemas sociales humanos, cuando las personas resuelven problemas compensan su conocimiento limitado al intercambiar información de manera directa o indirecta (consejos, experiencia, informes, noticias, etc.) con otros con los que interactúan. Esto pone de manifiesto la posibilidad de influir y ser influido por otros.

La interacción entre los empleados se da a través de redes de afiliación formales e informales [48]. Las redes de afiliación formales hacen referencia a los equipos (áreas, departamentos, proyectos, etc.) conformados por la dirección de la organización. Las redes formales se valoran por el respaldo institucional que reciben. Al ser incentivadas por el núcleo administrativo reciben mayor apoyo para su gestión. Sin embargo, no garantizan la empatía entre las partes. Por su parte, las redes de afiliación informales enfocan la red de lazos sociales que surgen en los empleados y que denotan relaciones 
de amistad, compañerismo, la relación de personas que comparten la ruta de transporte, que salen juntas a almorzar, etc. Las redes informales tienen la ventaja de fomentar la existencia de vínculos fuertes entre los empleados, los cuales refuerzan la comunicación, la confianza, la generación de ideas creativas, promueven la innovación y permiten que las organizaciones que operan en entornos altamente cambiantes respondan de manera flexible y dinámica [49].

Adicionalmente, la propuesta reconoce que todos los individuos tienen un determinado nivel de umbral de cambio que hace referencia a la susceptibilidad a adoptar una propuesta de acción. Diversas investigaciones han señalado que el umbral de cambio individual guarda estrecha relación con la interacción con el grupo [50]; es decir, si bien aspectos culturales, psicológicos o académicos pueden influir en la decisión de una persona de aceptar o no una propuesta de acción, existe un amplio nivel de relevancia en dicha decisión en la interacción que la persona tenga con otros miembros de la organización [51]. En el estudio de comportamientos sociales se encuentra evidencia de lo anterior en los altos niveles de influencia que ejercen ciertos líderes de opinión en las ideas o comportamientos de otros actores sociales.

Las organizaciones empresariales las conforman redes de afiliación formal e informal en las que existen nodos (personas) que, por diferentes razones, pueden ejercer altos niveles de influencia sobre otros con los que interactúan. El plural de la palabra redes busca denotar que no todos los empleados de la organización están conectados entre sí; es decir, no todos tienen interacción con todos, lo cual hace que emerjan distintas redes que interactúan unas con otras (figura 1).

La figura 1 presenta un ejemplo de una red que surge al interior de una organización, considerando las relaciones de afiliación informal. Este ejemplo es planteado por Krackhardt, quien muestra una red de amistad e indica con la forma de los nodos el departamento al que pertenecen los empleados, con el color los niveles jerárquicos y con un número la edad. Las relaciones de amistad son bidireccionales, es decir, reciprocas. Con las tres líneas circulares se propone la posibilidad de que las relaciones de amistad se dan preferentemente entre personas del mismo departamento y del mismo nivel jerárquico y, quizás, de una edad similar (todos ellos datos atributivos de los nodos). Sin embargo, en un

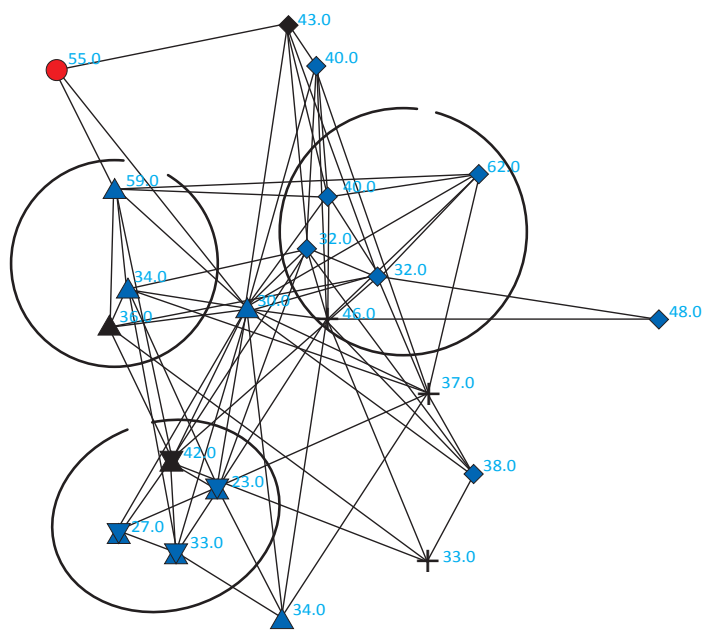

Figura 1. Red de amistad en una organización Fuente: [52], [53]

contexto empresarial unas personas pueden informar o influenciar preferentemente a otras y no al revés, lo que se evidencia con mayor claridad en la figura 2, en la cual se muestra una adaptación del modelo de un "Mundo Pequeño" de Watts y Strogatz en 1998.

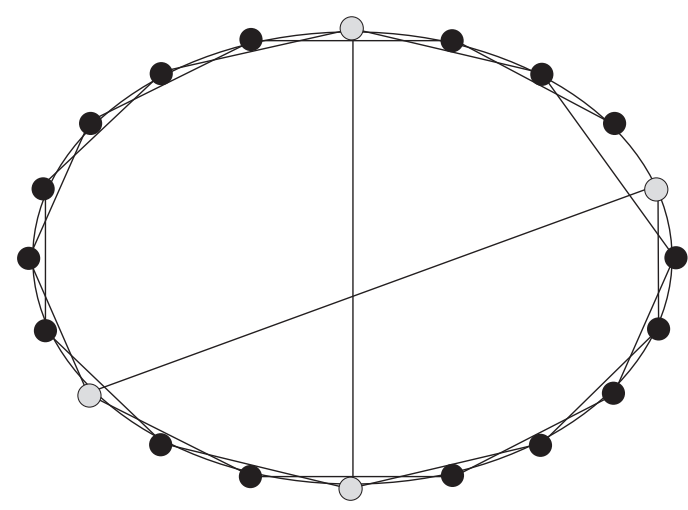

Figura 2 . Adaptación del modelo de un "Mundo Pequeño" Fuente: [53], [54]

Cada nodo tiene conexiones locales, pero en máximo tres pasos es posible alcanzar cualquier otro nodo del grafo. Al mismo tiempo, los cuatro nodos en gris acaparan el $80 \%$ de los caminos posibles entre los nodos de la red, lo cual permite transferir la información dado el nivel de influencia que hay entre los nodos en forma unidireccional. La investigación realizada por Freeman en 1979 
evidencia que existen tres criterios que permiten valorar el nivel de influencia que ejerce una persona (nodo) dentro de la empresa: grado, intermediación, cercanía [53]. En la figura 2, los nodos con mayor nivel de conexiones pueden estar relacionados con algunos de estos criterios.

El grado hace referencia al número de personas que dependen de un cargo específico, y con el que están directamente relacionadas. Una persona con alto grado significa que tiene una gran cantidad de empleados bajo su responsabilidad y, por tanto, tiene un alto potencial para influir en el comportamiento de la organización (en este caso el uso de ERP).

La intermediación es la frecuencia con la que una persona está en medio de la comunicación entre otras. Se considera central por la posibilidad de influir en el grupo al ocultar o distorsionar la información que transmite.

La cercanía es la medida con la que una persona está más cerca de los demás y, por tanto, no requiere de intermediarios para comunicarse. La cercanía se encuentra estrechamente relacionada con vínculos informales (e. g. amistad), y de allí el potencial para influir en las decisiones del equipo.

De acuerdo con lo anterior, la propuesta plantea que la internalización del uso de ERP en las actividades cotidianas de la empresa debería partir de la identificación de las personas que tienen el mayor potencial de influir sobre las demás, al considerar criterios de grado, intermediación y cercanía. De esta manera, la internalización de ERP puede iniciarse como un proceso de propalación de información acerca de las características, las ventajas y las limitaciones, entre otros aspectos de ERP, que circulan desde los nodos más influyentes hacia aquellos con los que interactúan.

Uno de las principales bondades de comprender las organizaciones como redes de afiliación formales e informes en las que el cambio surge a través de procesos coevolutivos es reconocer cómo, en la medida en que una persona con alto nivel de influencia facilite propagar la información acerca del uso de ERP, puede llegar a influir a otra u otras que, si bien no están tan conectadas como el anterior, sí tienen un alto nivel de conexiones. Desde esta perspectiva, el éxito en la internalización del uso de ERP se encuentra en facilitar el flujo de información desde los nodos o personas con mayor nivel de interacción hacia otros miembros de la red en los que disminuye el nivel de interacción.
La coexistencia de las redes formales e informales en el diseño de estructuras organizacionales y la implementación de proyectos genera amplios beneficios en el desempeño empresarial; sin embargo, los beneficios son mayores al combinar de manera adecuada los dos tipos de red. Las redes "semiformales" - como las denominó Biancanisugieren un mayor flujo de información dado que aprovechan el respaldo institucional de las redes formales soportadas en los altos niveles de confianza, creatividad, innovación y flexibilidad que generan las redes informales [54].

Con el fin de dar sentido a la situación actual de la organización, los miembros de la red buscan señales de fuentes formales e informales. Esto debido a que los comportamientos colectivos son en gran parte impulsados por las interpretaciones socialmente compartidas de acciones y eventos. Los altos niveles de desempeño que alcanzan las redes semiformales se encuentran relacionadas con la capacidad de procesamiento de información que las caracteriza [55].

Las redes semiformales surgen por decisiones del núcleo central de la organización, pero la integración de los empleados es voluntaria. Existen amplias alternativas para hacer operativas las redes semiformales. Nokia es pionero en su uso, pues anualmente los ejecutivos crean una hoja de ruta estratégica que comparten con los empleados a fin de motivar su integración en torno a objetivos o proyectos particulares, y formar equipos estratégicos por proyecto. Algunos de los miembros los nombran los ejecutivos, mientras que otros se unen voluntariamente [56]. Las personas asisten porque quieren compartir experiencia, conocimientos o aprender de lo que otros están haciendo.

Desde esta perspectiva, la resistencia al cambio a nivel individual es una situación que en algunos casos podría subsanarse en la medida en que la organización aproveche las bondades de la coevolución de las redes de afiliación semiformales que existen en la organización. Este concepto ha sido ampliamente estudiado en la ciencia de redes [55] [55], y plantea la necesidad de redes ampliamente conectadas como mecanismo facilitador para el surgimiento de comportamientos colectivos en un sistema.

Sin embargo, la investigación al respecto en organizaciones empresariales se encuentra en etapas tempranas; a la fecha no existe un modelo o una propuesta que facilite aprovechar la coevolución de 
las redes inter e intraorganizacionales. La presente investigación se encuentra en fase conceptual y presenta limitaciones relacionadas con la carencia de investigación empírica que permita su validación con información del mundo real.

Con ayuda del programa Net Logo, desarrollado por U. Wilensky en 1999, se realizan simulaciones que permitan observar la efectividad del flujo de información a partir de nodos (personas) que, por diferentes razones, pueden ejercer altos niveles de influencia sobre otros con los que interactúan.

Los resultados demuestran cómo, a medida que pasa el tiempo, la información se propaga entre todas las personas al interior de la organización de modo similar a lo que ocurre con un virus o un rumor. El éxito está en garantizar este flujo de información en todo momento y en que las personas escogidas como fuentes estén alineadas con las estrategias de la dirección y vayan en pro de lo que se quiere lograr.

En esta línea se encuentra la simulación Rumor Mill, desarrolla en Net Logo [56], en la que se muestra cómo una idea - en este caso un rumor- se extiende cuando una persona lo comenta con unos de los vecinos con los que interactúa, hasta que toda la población adquiere la información (figura $3 a, b$, c). Esta simulación muestra la relevancia de la cercanía en la posibilidad de influir en otros con los que se interactúa.

También se resalta la simulación Virus desarrollada en Net Logo [58], en la que se reconoce la existencia de agentes con capacidad de infectar a otros (color rojo), agentes inmunes al virus (color gris), y la población sana susceptible de infectarse (color verde). La simulación muestra la forma como se programa el virus en la medida en que los agentes interactúan (figura $4 \mathrm{a}, \mathrm{b}$ ). La relevancia para la presente investigación es el reconocimiento de que todos los individuos tienen un nivel de umbral de

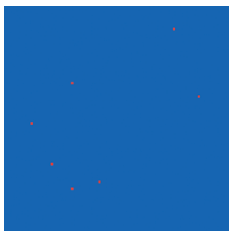

a.

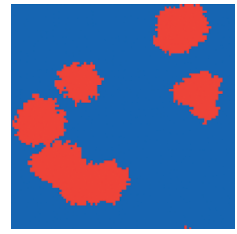

b.

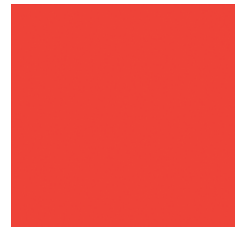

c.
Figura 3. Evolución en la propagación de un rumor Fuente: [57] cambio, pero que, a pesar de esto, el virus logra convertirse en un comportamiento colectivo, tal como se busca con la internalización del cambio desde el uso de ERP.

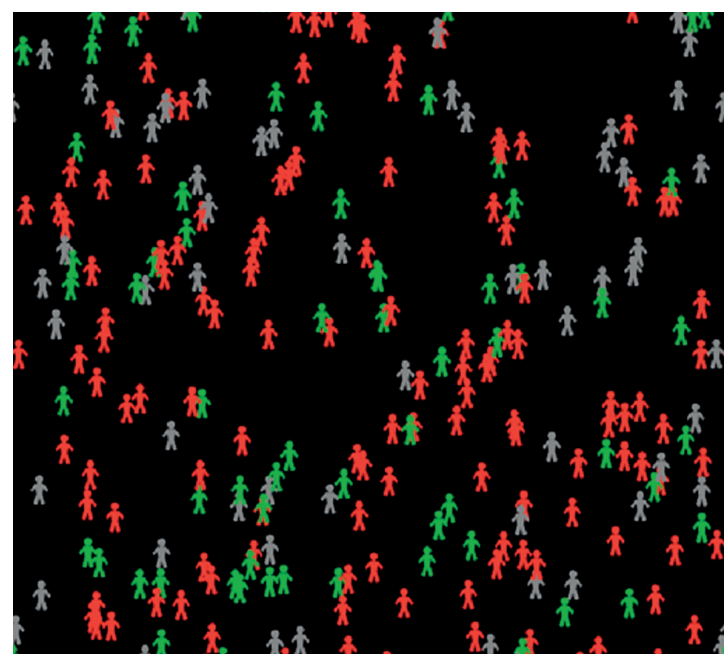

Figura 4a. Virus Net Logo

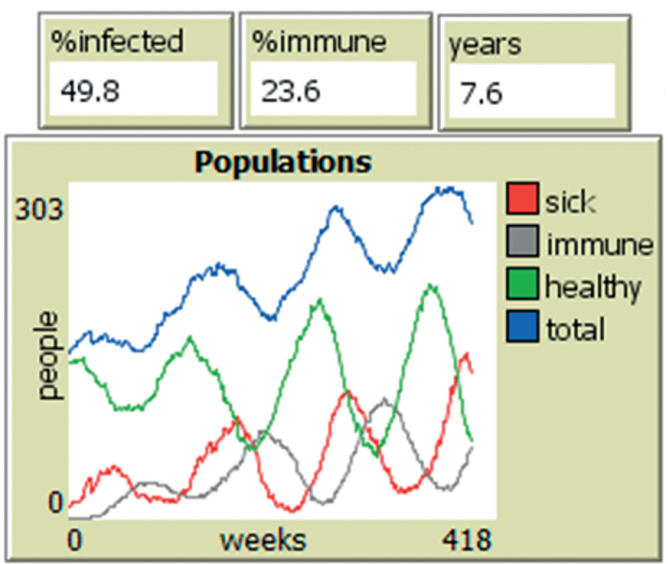

Figura 4b. Evolución en la propagación de un virus Fuente [58]

\section{Discusión}

Las redes internas pueden generar intercambios positivos y beneficiosos en los recursos interpersonales como, por ejemplo, la satisfacción laboral (el intercambio de los recursos aumenta la percepción de apoyo del compañero de trabajo) y el arraigo en el empleo (relacionado con el sentimiento de vinculación y gusto por el trabajo). Las redes informales tienen la ventaja de fomentar la existencia 
de vínculos fuertes entre los empleados, los cuales refuerzan la comunicación, la confianza, la generación de ideas creativas, promueven la innovación y permiten a las organizaciones que operan en entornos altamente cambiantes responder de manera flexible y dinámica.

En las organizaciones empresariales, y de manera amplia en los sistemas sociales humanos, cuando las personas resuelven problemas compensan su conocimiento limitado con el intercambio de información de manera directa o indirecta (consejos, experiencia, informes, noticias, etc.) con aquellos con los que interactúan. Por tanto, es necesario determinar y discutir cómo lograr que este intercambio de información se dé con las personas correctas y se evite compartir información errónea.

Se evidencian tres criterios que permiten valorar el nivel de influencia que ejerce una persona (nodo) dentro de la empresa: grado, intermediación y cercanía. La internalización del uso de ERP en las actividades cotidianas de la empresa debería partir de identificar las personas que tienen el mayor potencial de influir sobre las demás, de acuerdo con criterios de grado, intermediación y cercanía. Ahora bien, entra en discusión la pregunta: ¿Cómo logramos identificar correctamente a estas personas?

Si bien la presente propuesta se encuentra en proceso, puede escalarse a la comprensión de otros fenómenos en sistemas sociales humanos tales como el éxito empresarial. Lo anterior debido a que proporciona claridad de las ventajas en las interacciones humanas y del flujo de la información, pues a medida que dichas interacciones son fuertes existen nodos potenciales que impulsan una idea.

La comprensión del cambio como proceso de coevolución en redes formales e informales da pie a la opción de discutir y repensar la forma como están organizadas las empresas. De tal manera, se plantea la posibilidad de pasar de estructuras rígidas y con fallas de comunicación a unas más flexibles, con mayor conexión en las redes y mejor flujo de información.

\section{Conclusiones}

El término evolución es sinónimo de cambio, y son términos implícitamente relacionados. La coevolución hace referencia a los cambios recíprocos entre especies interactuantes. Al interior de las organizaciones empresariales son los trabajadores quienes se relacionan y crean redes formales y no formales de comunicación, las cuales permiten el flujo continuo de información y, de esta manera, se logra la internalización de ERP.

El presente estudio parte de la premisa según la cual el uso generalizado de ERP en las actividades cotidianas de la empresa que decida implementarlo puede comprenderse como la emergencia de un comportamiento colectivo en un sistema caracterizado por altos niveles de interacción interna y externa.

La propuesta se soporta en tres aspectos: a) las organizaciones son redes de interacción que se dan por relaciones laborales o informales, surgen de las relaciones sociales de los empleados y denotan compañerismo, amistad, etc.; b) la interacción explica la posibilidad de influir y ser influido (coevolución); c) la coevolución explica el surgimiento de comportamientos colectivos tales como el uso generalizado de ERP en las organizaciones empresariales que decidan implementarlo, pues se da un proceso de reciprocidad entre las partes que permite un mejor flujo de la información.

Todos los individuos tienen una determinada resistencia al cambio referente a la susceptibilidad a adoptar una propuesta de acción. Diversas investigaciones han señalado que el umbral de cambio individual guarda estrecha relación con la interacción con el grupo [44]; es decir, si bien aspectos culturales, psicológicos y académicos pueden influir en la decisión de una persona de aceptar o no una propuesta de acción, existe un amplio nivel de relevancia en dicha decisión en la interacción que la persona tenga frente a otros miembros de la organización.

El éxito en la internalización del uso de ERP se da en facilitar el flujo de información desde los nodos o personas con mayor nivel de interacción hacia otros miembros de la red en los que disminuye de manera progresiva el nivel de interacción.

Si bien esta propuesta está dirigida a la implementación de ERP, es extensible a todos aquelos proyectos que implican un cambio y un flujo de información a todos los miembros de una organización.

\section{Referencias}

[1] E. Kimberling, "ERP failures and lawsuits: It's not just for the tier I ERP vendors," Panorma Cosulting Solutions, en. 2011, pp. 1 [En línea]. Disponible en: 
http://panorama-consulting.com/erp-failures-andlawsuits-its-not-just-for-the-tier-i-erp-vendors/

[2] C. Leyh, "Critical success factors for ERP projects in small and medium-sized enterprises-the perspective of selected German smes," en Computer Science and Information Systems (FedCSIS), Varsovia, Polonia: IEEE, 2014 pp. 1181-1190. doi: 10.15439/2014F243

[3] R. Kouki, D. Poulin y R. Pellerin. ERP assimilation challenge: an integrative framework for a better post-implementation assimilation. Interuniversity research center on enterprise networks, logistics and transportation (Cirrelt), Unversite Laval, Québec: dic. 2006, pp. 1-41. Disponible en: https://www.cirrelt.ca/ DocumentsTravail/2006/DT-2006-DP-1.pdf

[4] T. Davenport, "Putting the enterprise into the enterprise system”, Harvard Business Review, jul-agos. 1998, pp. 121-131. [En línea]. Disponible en: https://hbr.org/1998/07/putting-the-enterprise-into-the-enterprise-system

[5] M. Ağaoğlu, S. Yurtkoru y A. K. Ekmekçi, “The effect of ERP implementation CSFS on business performance: an empirical study on users' perception", Elsevier, vol. 210, pp. 35-42, 2015. doi: https://doi. org/10.1016/j.sbspro.2015.11.326

[6] S. Shari y P. B. Seddon, "A comprehensive framework for classifying the benefits of ERP systems", en Americas Conference on Information Systems (Amcis), vol. 1, no. 1, 2000, pp. 1-11. [En línea]. Disponible en: http://citeseerx.ist.psu.edu/viewdoc/download? doi=10.1.1.361.5523\&rep $=$ rep $1 \&$ type $=$ pdf

[7] S. Chou y Y. C. Chang, "The implementation factors that influence the ERP (enterprise resource planning) benefits", Decision Support Systems, vol. 46, n. ${ }^{\circ}$ 1, dic. 2008, pp. 149-157. [En línea]. Disponible en: https://dl.acm.org/ citation.cfm?id=1464564

[8] V. Tamturk, “ERP implementation failure: MillerCoors sues HCL for \$100M”, Cms-connected.com, mar. 31 2017. [En línea]. Disponible en: http://www. cms-connected.com/News-Archive/March-2017/ ERP-SAP-Implementation-Failure-MillerCoorsSues-HCL-for-\$100M

[9] J. Fruhlinger y T. Wailgum, "15 famous ERP disasters, dustups and disappointments”, Cio.com, jul. 2017. [En línea]. Disponible en: https://www.cio.com/ article/2429865/enterprise-resource-planning/enterprise-resource-planning-10-famous-erp-disasters-dustups-and-disappointments.html

[10] B. Stock, “Top six ERP implementation failures", 360cloudsolutions.com, 2017. [En línea]. Disponible en: http://www.360cloudsolutions.com/top-sixerp-implementation-failures/

[11] Wayferry.com, "Wayferry case study: the curious case of cubic corporation and the missing RoI", Wayferry.com, jul. 2017. [En línea]. Disponible en: https://www.wayferry.com/tags/case\%20studies
[12] K. Zhu, K. Kraemer y S. Xu, "The process of innovation assimilation by firms in different countries: a technology diffusion perspective", Managment Science, vol. 52, no. 10, pp. 1557-1556, 2006. doi: 10.1287/mnsc.1050.0487

[13] C. Jiménez, "Propuesta para la valoración tecnológica desde la perspectiva de la síntesis evolutiva moderna”, tesis doctoral, Universidad Nacional de Colombia, Bogotá, 2015, pp. 97-162. [En línea]. Disponible en: http://www.bdigital. unal.edu.co/53221/

[14] E. Andrade, "Analogías entre las teorías de la materia, la evolución biológica y la sociedad", en $\mathrm{Me}$ morias del seminario de ciencia teórica 1994-1996, Bogotá: Instituto de Ciencias Naturales, Editorial Universidad Nacional de Colombia, 1996, pp. 101-123. [En línea]. Disponible en: http://www. academia.edu/2630885/ ANALOGIAS_MATERIA_-EVOLUCION_-SOCIEDAD_ACCEFN_Colecci\%C3\%B3n_Memorias_No_8_1996

[15] J. N. Thompson. The coevolutionary process. Chicago: Chicago University, 1982, pp. 50-65.

[16] E. D. Brodie, "Predator-prey arms races", BioScience, vol. 49, no. 7, 1999, pp. 557-568. doi: https://doi. org/10.2307/1313476

[17] L. V. Valen. A new evolutionary law, Chicago: Chicago University, 1973. [En línea]. Disponible en: http://ebme.marine.rutgers.edu/HistoryEarthSystems/HistEarthSystems_Fall2010/VanValen\%20 1973\%20Evol\%20\%20Theor\%20.pdf

[18] J. Dimmick, "Sociocultural evolution in the communication industries", SAGE, vol. 13, no. 3, 1986, pp. 473-508. [En línea]. Disponible en: http:// journals.sagepub.com/doi/pdf/10.1177/0093650 86013003011

[19] M. T. Hannan y J. H. Freeman, "The population ecology of organizations", American Journal of Sociology, vol. 82, no. 5, pp. 929-964, 1977. [En línea]. Disponible en: http://www.jstor.org/stable/2777807

[20] H. E. Aldrich. Organizations and environments. Stanford: Stanford University, 2008, pp. 26-54.

[21] K. E. Weick, "The social psychology of organizing", M@n@gement, vol. 18, 2015-2, pp. 189-193. [En línea]. Disponible en: http://www.cairn.info/revue-management-2015-2-page-189.htm

[22] B. McKelvey. Organizational systematics: taxonomy, evolution. Los Angeles: University of California Press, 1982, pp. 80-99.

[23] K. R. Andrews. The concept of corporate strategy. Berkeley: University of California, 1971, pp. 195210.

[24] J. Child, "Organization structure, environment and performance: The role of strategic choice", Sociology, vol. 6, no. 1, pp. 1-22, en. 1972. [En línea]. Disponible en: http://www.jstor.org/stable/42851133 
[25] J. P. Murmann, “The coevolution of industries and important features of their environments", Organization Science, vol. 24, no. 1, feb. 2013, pp. 58-78. [En línea]. Disponible en: https://pdfs.semanticscholar.org/6dca/ 132b3f4498486cdb4e68a8bf702a2d74c2b4.pdf

[26] R. R. Wiggins y T. W. Ruefli, "Schumpeter's ghost: is hypercompetition making the best of times shorter?", Strat. Mgmt. J., vol. 26, pp. 887-91, 2005. doi: 10.1002/smj.492

[27] R. A. D’Aveni, G. B. Dagnino y K. G. Smith, “The age of temporary advantage”, Strat. Mgmt. J., vol. 31, no. 13, pp. 1371-1385, dic. 2010. doi: 10.1002/smj.897

[28] A. Y. Lewin, C. P. Long y T. N. Carroll, "The coevolution of new organizational forms," Organization Science, vol. 10, pp. 535-550, sep. 1999. [En línea]. Disponible en: http://pubsonline.informs.org/doi/ abs/10.1287/orsc.10.5.535

[29] J. P. Murmann. Knowledge and competitive advantage: the coevolution of firms, technology, and national institutions. Nueva York: Cambridge University Press, 2003, pp. 154-172. doi: https://doi. org/10.1017/CBO9780511510953

[30] J. A. Yates, "Co-evolution of information-processing technology and use: interaction between the life insurance and tabulating industries", The Business History Review, vol. 67, no. 1, pp. 1-51, 1993. [En línea]. Disponible en: http://www.jstor.org/stable/3117467

[31] R. R. Nelson, "The co-evolution of technology, industrial structure, and supporting institution", Industrial and Corporate Change, vol. 3, pp. 47-63, en. 1994. [En línea]. doi: https://doi.org/10.1093/ icc/3.1.47

[32] L. Rosenkopf y M. Tushman. Evolutionary dynamics of organizations. Nueva York: Oxford University Press, 1994, pp. 57-83.

[33] A. D. Henderson e I. Stern, "Selection-based learning: the coevolution of internal and external selection in high-velocity environments", Administrative Science Quarterly, vol. 49, no. 1, pp. 39-75, mar. 2004. [En línea]. Disponible en: http://www.jstor. org/stable/4131455

[34] J. Funk, "The co-evolution of technology and methods of standard setting: the case of the mobile phone industry", J. Evolutionary Econom, vol. 19, n. ${ }^{\circ}$ 1, pp. 73-93, 2009. [En línea]. Disponible en: https://link.springer.com/article/10.1007/ s00191008-0108-6

[35] B. R. Johnson y S. K. Lam, "Self-organization, natural selection, and evolution: cellular hardware and genetic software", Bioscience, vol. 60, no. 11, pp. $879-885$, dic. 2010. doi: https://doi.org/10.1525/ bio.2010.60.11.4
[36] D. Plikynas, "A virtual field-based conceptual framework for the simulation of complex social systems," Journal of Systems Science and Complexity, vol. 23, n. ${ }^{\circ} 2$, pp. 232-248, abr. 2010. doi: https://doi. org/10.1007/s11424-010-7239-1

[37] P. Phister, "Cyberspace: the ultimate complex adaptive system", Int C2 J, vol. 4, no. 2, pp. 1-33, 2011. [En línea]. Disponible en: http://www.dodccrp.org/files/ IC2J_v4n2_03_Phister.pdf

[38] G. Maione y D. Naso, "Adaptation of multi-agent manufacturing control by means of genetic algorithms and discrete event simulation", en Proceedings of the IEEE International Conference on Systems, Man and Cybernetics, vol. 4, pp. 529-534, Univ. degli Studi di Lecce, Dip. Di Ingegneria dell'Innovazione. [En línea]. doi: 10.1109/ICSMC.2002.1173342

[39] M. Brezocnik y J. Balic, "A genetic-based approach to simulation of self-organizing assembly", Robotics and computer-integrated manufacturing, vol. 17, no. 1-2, pp. 113-120, 2001. [En línea]. Disponible en: http://www.sciencedirect.com/science/article/pii/ S0736584500000442

[40] K. Ueda, J. Vaario y K. Ohkura, "Modelling of biological manufacturing systems for dynamic reconfiguration", CIRP Annals-Manufacturing Technology, vol. 46, no. 1, pp. 343-346, 1997. doi: https://doi. org/10.1016/S0007-8506(07)60839-7

[41] L. A. Montoya, "Gestión de sistemas de integración empresarial desde una perspectiva biológica”, tesis doctoral, Departamento de Ciencias Económicas, Universidad Nacional de Colombia, Bogotá: 2010, pp. 1-220. [En línea]. Disponible en: http://www. bdigital.unal.edu.co/2140/1/GESTI\%C3\%93N_ DE_SISTEMAS_DE_INTEGRACI\%C3\%93N_EM PRESARIAL.pdf

[42] I. A. Otálora, "Pertinencia del fundamento biológico para el mejoramiento de la productividad en los sistemas de manufactura", tesis de maestría, Departamento de Ingeniería Industrial, Universidad Nacional de Colombia, Bogotá: 2014, pp. 1-152. [En línea]. Disponible en: http://libros.duhnnae.com/2017/ jun3/149677581433-Pertinencia-del-fundamento-biologico-para-el-mejoramiento-de-la-productividad-en-los-sistemas-de-manufactura-62-Ingenieria-y-operaciones-afines-.php

[43] B. McKelvey, "Toward a complexity science of entrepreneurship“, Journal of Business Venturing, vol. 19, n. 3 pp. 313-341, may. 2004. doi: https://doi. org/10.1016/S0883-9026(03)00034-X

[44] R. D. Stacey, "The science of complexity: an alternative perspective for strategic change processes", Strategic Management Journal, vol. 16, pp. 477-495, sep. 1995. [En línea]. Disponible en: 
http://citeseerx.ist.psu.edu/viewdoc/ download?doi=10.1.1.465.3554\&rep=rep1\&type $=$ pdf

[45] J. M. Acosta, L. Y. Gualdrón y L. E. Bohórquez, “Organizational structures and adaptation to the changing conditions of the environment: challenges and implications", Ingeniería Solidaria, vol. 23, no. 23, 2017. doi: https://doi.org/10.16925/in.v23i13.1983

[46] M. S. Granovetter, “The strength of weak ties”, American Journal of Sociology, vol. 78, pp. 1360-1380, may. de 1973. doi: https://doi.org/10.1086/225469

[47] S. Biancani, D. A. McFarland y L. Dahlander, "The semiformal organization", Organization Science, vol. 25, pp. 1306-1334, feb. 2014. doi: https://doi. org/10.1287/orsc.2013.0882

[48] D. J. Watts. Seis grados de separación, la ciencia de las redes en la era del acceso. Barcelona: Ed. Paidós Ibérica: 2006, pp. 386.

[49] A. L. Barabási. "From society to the web", Cambridge, MA: Ed. The New Science of Networks, Perseus Group Book, 2002, pp. 307-321.

[50] D. Krackhardt, "Assessing the political landscape: structure, cognition, and power in organizations", Administrative Science Quarterly, no. 35, pp. 342-69, 1990. [En línea]. Disponible en: https://pdfs.semanticscholar.org/4d27/eb71703defa42c2402b9681592 941a285e5f.pdf

[51] J. L. Molina, "La ciencia de las redes," AACTE, vol. 25, pp. 36-42, jun. 2004. [En línea]. Disponible en: http://revista-redes.rediris.es/recerca/jlm/ars/ciencia.pdf
[52] D. J. Watts y S. H. Strogatz, "Collective dynamics of 'small-world' networks”, Nature, vol. 393, pp. 440442, jun. 1998. doi: 10.1038/30918

[53] L. C. Freeman, "Centrality in social networks conceptual clarification", Social Network, vol. 1, p. 215 239, 1979. [En línea]. Disponible en: http://citeseerx.ist.psu.edu/viewdoc/download?doi=10.1.1. 320.5551\&rep $=$ rep $1 \&$ type $=$ pdf

[54] B. G. King, E. S. Clemens y M. Fry, "Identity realization and organizational forms: differentiation and consolidation of identities among Arizona's charter schools", Organ. Sci., vol. 22, no. 3, pp. 554572, 2011. doi: http://pubsonline.informs.org/doi/ abs/10.1287/orsc. 1100.0548

[55] S. E. Seibert, L. D. Sargent, M. L. Kraimer y K. Kiazad, "Linking developmental experiences to leader effectiveness and promotability: the mediating role of leadership selfefficacy and mentor network", Pers. Psychol., vol. 70, no. 2, pp. 357 -397, 2017. doi: http://onlinelibrary.wiley.com/doi/10.1111/peps. $12145 /$ full

[56] U. Wilensky. NetLogo. Evanston, IL: Northwestern University, 1999, pp.1.

[57] U. Wilensky, "NetLogo Rumor Mill model", en Center for Connected Learning and Computer-Based Modeling, Evanston, IL: Northwestern University, 1997.

[58] U. Wilensky, "NetLogo," en Center for Connected Learning and Computer-Based Modeling Northwestern University, Evanston, IL: 1999. 